\title{
Introduction: immunopathology and the promise of stem cell research
}

\author{
Olivier Preynat-Seauve • Jean Villard
}

Received: 26 April 2011 / Accepted: 17 May 2011 / Published online: 28 May 2011

(C) Springer-Verlag 2011

During the last few years, stem cell technology has elicited a great deal of fascination, hope and controversy as have done other cell research programs. The primary aim of developing stem cells for medical application is to regenerate tissues and repair failing organs. In addition, stem cell research can be viewed as a key to addressing and understanding many of the most fundamental and basic questions in clinical biology. A better insight into early cell development will provide answers to the question why cells behave abnormally and generate pathologies such as cancer or autoimmune and degenerative diseases. The huge interest focussing on this research and its potentially therapeutic applications are evident from the large investment made by companies, governments and universities worldwide.

In October and November 2010, the two first human trials of cell therapy by embryonic stem cell-derived injections for spinal cord injury and non-hereditary forms of blindness were approved by the FDA. The source of stem cells, however, remains a subject of debate. Taking into account genetic and immunological criteria, stem cells can be derived from either of the following:

1. An individual that is genetically unrelated to the recipient. This applies to cells like embryonic stem cells.

This article is published as a part of the special issue on Immunopathology of Pluripotent Stem Cell Transplantation [33:6].

O. Preynat-Seauve

Laboratory of Experimental Cell Therapy,

Geneva University Hospital and Medical School,

Geneva, Switzerland

J. Villard $(\bowtie)$

Transplant Immunology Unit, Division of Immunology and Allergy and Division of Laboratory Medicine, Geneva University Hospital and Medical School,

4, rue Gabrielle-Perret-Gentil,

1211 Geneva 14, Switzerland

e-mail: jean.villard@hcuge.ch
2. Adult cells that have been reprogrammed to induce pluripotent stem cells (iPS). The advantage of iPS is that they are derived from adult tissues, thus obviating the use of embryos. The origin of the adult tissue to prepare iPS can theoretically be the patient itself, leading to the situation of autologous transplantation. Unfortunately, this is offset by some drawbacks that, for the time being, preclude their use in procedures of human stem cell therapy. The use of transduction vectors like retroviruses could be associated with insertional mutagenesis and the insertion of pluripotent genes like the oncogene MIC could theoretically be linked to tumorigenesis. Also, from a practical point of view, the preparation of iPS of a clinical grade suitable for transplantation in patients could be difficult to achieve for reasons of time and cost effectiveness. Different methods will have to be set up, leading to homogeneous and reproducible cell populations that do not form teratoma or other cancers. Therefore, the use of stem cells derived from embryos or the creation of banks of stem cells generated in good manufacturing practice laboratories would signify a genetic mismatch between donor's and recipient's cells and serious problem of transplant rejection by the immune system. Despite the potential of human embryonic stem (ES) cells, important issues surrounding immunogenicity subsist, and strategies to avoid rejection remain largely untested. In addition, even if iPS derived from the patients would constitute eventually a perfect immunological match, the risk of rejection by inflammation and by the innate immune system activated by the transplantation procedure must not be taken lightly.

The special reviews issued by the Seminars in Immunopathology are intended to summarize current knowledge on immunological problems with regards to 
the transplantation of stem cells or their progenitors. In the first review, obtaining stem cells for regenerative medicine is extensively discussed by Olivier Preynat-Seauve and Karl-Heinz Krause. Several types of stem cells have been identified and isolated from embryos, foetus or adult organs, and each distinct type of stem cells harbors different properties, providing multiple strategies, while immunological considerations associated with their use are highlighted. Casmir de Rham and J. Villard summarize the genetic and immunological barriers to human ES cell transplantation. These are: blood groups, MHC and minor antigens that have to be crossed for a successful transplantation. One of the principal difficulties in cell and tissue transplantation is the implantation step. Engraftment and persistency of cells are impeded by several adverse processes like apoptosis, anoikis or necrosis. Ian Copland and Jacques Galipeau review these crucial difficulties, taking as an example mesenchymal stem cells (MSC) that are currently used in clinical trials for different disorders ranging from heart disease to graft-versus-host disease in pediatric patients. This is, as far as we know, one of the first reviews on this critical topic. The potential use of MSC for tissue repair and immunotherapy has been clearly demonstrated during the past decade. MSC can exhibit immunomodulatory functions that could be beneficial for cell therapy. Ilaria Marigo and Francesco Dazzi have contributed a state of-the-art review on this important topic.

Immunogenicity of undifferentiated pluripotent stem cells could be different from the lineage derived from them. Susan Kadereit reviews this highly important aspect of stem cell biology. Philippe Capetian, Maté Döbrössy, Christian Winkler, Marco Prinz and Guido Nikkhah point out the implications of grafting neural stem cells into the central nervous system. The procedure has been performed in several animal models and also in a limited number of small human clinical studies. The authors relate their experiments with allograft cells in the absence of immunosuppressive agents and the role of immunologically privileged site like CNS as compared to other engraftment sites in the body. Chad Tang and Micha Drukker focus on a very interesting topic, that of propagation and nonphysiological differentiation of pluripotent stem cells which can induce the expression of aberrant antigens. This may apply to allogenic pluripotent stem cells but also for iPS with new antigens resulting from long-term maintenance in vitro.

The article by Senju Satoru, Yusuke Matsunaga, Satoshi Fukushima, Tokumori Ikeda and Yasuharu Nishimura assesses the suitability of pluripotent stem cells for generating dendritic cells (DC) and providing an unlimited source of DC for potential use in anti-cancer vaccine therapy. The authors describe methods of differentiation into functional DC from pluripotent stem cells of mice and human origins. Nicholas Zavazava reviews the use of human ES cells to generate an alternative source of hematopoietic stem cells for possible use in cell therapy in medical conditions including hematopoietic stem cell malignancies, immunodeficiency and autoimmune diseases. He discusses the immunological properties of ES derived from hematopoietic progenitors and from other tissue.

One major problem encountered in the clinical application of stem cells is the risk of tumor formation. Ralf Dressel highlights recent data on the tumorigenicity of pluripotent stem cells in autologous, allogenic and xenogenic immunodeficient hosts. The effect of immunosuppresive treatment on tumor development is also discussed. Finally, Outi Hovatta examines the critical issue of clinical applications of stem cells and their linage. Stem cells have to be cultured according to good manufacturing practice and a quality control system to minimize the risks. The screening of donor cells for human pathogens, the selection of xeno-free chemicals and meticulously validated laboratory procedures are crucial steps to implement and prove the procedure's safety.

Undoubtedly, stem cells research will continue to offer tremendous promise along with the possibility of generating products that could revolutionize the treatment of disease and the regeneration of damaged tissues. This optimistic outlook needs, however, to be tempered owing to the plethora of ethical, legal, political and financial challenges that face this research. Some of these are certainly of an immunological nature and will have to be taken into account and solved, at least in part, to guarantee the success of human stem cell therapy. Meetings that provide the opportunity for interaction between scientists involved in stem cell research and those active in transplant immunology are ideal for considering and finding solutions to the serious issue represented by the rejection of the transplanted tissues. We hope that the current issue of the Seminars in Immunopathology will contribute to the joining of forces between basic scientists and clinicians with a view to obtain the best possible results in stem cell research for clinical application.

Acknowledgments JV and O.PS-are supported by the Swiss National Foundation for Research (CRSI33- 125408) and the Swiss Parkinson Foundation.

JV and O.PS are members of the Prometheus Consortium (Pluripotent stem cells for Therapy of Parkinson's Disease: A multidisciplinary and translational consortium). 